\title{
BMJ Open Long-chain omega-3 fatty acid serum concentrations across life stages in the USA: an analysis of NHANES 2011-2012
}

\author{
Rachel A Murphy (D , , ${ }^{1,2}$ Prasad P Devarshi, ${ }^{3}$ Shauna Ekimura, ${ }^{3}$ Keri Marshall, ${ }^{3}$ \\ Susan Hazels Mitmesser ${ }^{4}$
}

To cite: Murphy RA,

Devarshi PP, Ekimura S, et al. Long-chain omega-3 fatty acid serum concentrations across life stages in the USA: an analysis of NHANES 2011-2012. BMJ Open 2021;11:e043301. doi:10.1136/ bmjopen-2020-043301

- Prepublication history and additional supplemental material for this paper are available online. To view these files, please visit the journal online (http://dx.doi.org/10.1136/ bmjopen-2020-043301).

Received 04 August 2020 Revised 18 November 2020 Accepted 25 April 2021

Check for updates

(c) Author(s) (or their employer(s)) 2021. Re-use permitted under CC BY-NC. No commercial re-use. See rights and permissions. Published by BMJ.

${ }^{1}$ BC Cancer Research Centre, Vancouver, British Columbia,

Canada

${ }^{2}$ School of Population and Public Health, The University of British Columbia, Vancouver, British Columbia, Canada

${ }^{3}$ Pharmavite LLC, Mission Hills, California, USA

${ }^{4}$ Science and Technology,

Pharmavite LLC, Mission Hills, California, USA

Correspondence to

Dr Rachel A Murphy;

rachel.murphy@ubc.ca

\section{ABSTRACT}

Objective To determine reference ranges of circulating long-chain (LC) omega-3 fatty acids: eicosapentaenoic acid (EPA), docosapentaenoic acid (DPA) and docosahexaenoic acid (DHA) in a nationally representative population of Americans. To provide context, serum concentrations of LC omega-3 were compared with concentrations associated with consuming the recommended amount of EPA and DHA by the Dietary Guidelines for Americans (DGA) and the Omega-3 Index (EPA+DHA).

Design Cross-sectional population-based study.

Setting The National Health and Nutrition Examination Survey 2011-2012 cycle.

Participants Participants with fatty acids measured in serum: 945 children, age $3-19$ years, and 1316 adults, age 20 and older.

Main measure Serum EPA, DPA, DHA and sum of LC omega-3 fatty acids expressed as per cent of total fatty acids.

Results Among children, mean (SE) serum concentrations of EPA, DHA and omega-3s were $0.28 \%$ (0.01), $1.07 \%$ (0.02) and $1.75 \%(0.03)$. Among adults, mean (SE) of EPA, DHA and omega-3s were $0.61 \%(0.02), 1.38 \%(0.05)$ and $2.43 \%(0.08)$, all of which were significantly higher than corresponding serum fatty acid concentrations in children $(p<0.001)$. Despite recommendations for higher intake, pregnant and/or breastfeeding women had mean (SE) EPA, DHA and LC omega- 3 concentrations of $0.34 \%(0.07)$, $1.52 \%(0.08)$ and $2.18 \%(0.15)$, which were comparable to women of childbearing age; $p=0.17, p=0.10$ and $p=0.73$. Over $95 \%$ of children and $68 \%$ of adults had LC omega- 3 concentrations below those associated with the DGA recommendation. Approximately $89 \%$ of adults had an Omega-3 Index in the high cardiovascular risk category. Conclusions Contemporary reference ranges for circulating LC omega-3s are critical for setting public health recommendations. Our findings show the need for continued emphasis on regular consumption of LC omega-3s among Americans, particularly considering the importance of LC omega-3s in cardiovascular health, brain health and development throughout life.

\section{BACKGROUND}

Consumption of seafood rich in long-chain (LC) omega-3 polyunsaturated fatty acids (omega-3 PUFAs), from seafood and dietary

\section{Strengths and limitations of this study}

This study is one of the few to provide reference range data on circulating LC omega-3 fatty acids, a biomarker of LC omega-3 nutritional status using data that are representative of the children (three and older) and adults in the US population.

- Strengths of the study include the study design that allows inference to the general population in the US, inclusion of multiple life stages (children, adolescents, adults, seniors and pregnant or breastfeeding women) and serum biomarker measurement of LC omega-3 fatty acid intake.

- Limitations of the study include the lack of information on long-term LC omega-3 fatty acid intake, and possible shifts in diet and LC omega- 3 status since the time of measurement in 2011-2012.

supplements, is recommended as part of a healthy diet. ${ }^{12}$ A large body of evidence has shown that dietary patterns, including high consumption of LC omega-3 PUFAs eicosapentaenoic acid (EPA) and docosahexaenoic acid (DHA), both from diet and supplementation, are associated with decreased risk of cardiovascular disease, ${ }^{3}$ type 2 diabetes, ${ }^{4}{ }^{5}$ lower overall mortality ${ }^{6}$ and lower blood pressure. ${ }^{78}$ Strong evidence also supports a positive relationship between maternal dietary intake of EPA and DHA and neurodevelopment among infants. ${ }^{910}$ Further research also supports the role of EPA and DHA in neuropsychological health, such that EPA and DHA supplementation is considered as a part of integrative therapies for depressive disorder by the American Psychiatric Association. ${ }^{11}$

The most recent Dietary Guidelines for Americans (DGA 2010-2015 and 2015-2020) recommends that individuals two and older consume 8 ounces per week of a variety of seafood to provide approximately $250 \mathrm{mg}$ of EPA and DHA per day. ${ }^{2}$ Consuming this amount is associated with reduced cardiac death in those with and without preexisting cardiovascular disease. ${ }^{2}$ Similarly, 
the American Heart Association has recommended consuming 1-2 seafood meals per week to reduce the risk of congestive heart failure, coronary heart disease, ischaemic stroke and sudden cardiac death. ${ }^{12}$ Women who are pregnant or breastfeeding are encouraged to consume up to 12 ounces per week to obtain enough omega-3 in their diet to support infant health, ensuring they consume at least $300 \mathrm{mg} /$ day EPA+DHA, of which $\geq 200 \mathrm{mg}$ /day should be DHA specifically. ${ }^{213}$

Despite the health benefits of omega-3s, data from the National Health and Nutrition Examination Survey (NHANES) clearly shows that dietary intake of omega-3s in Americans is low and consistently well below the DGA recommendation across all age groups, gender and race/ ethnicity. ${ }^{214-16}$ In 2015, the DGA Committee reviewed data from What We Eat in America/NHANES and found $90 \%$ of Americans were below the recommended intake of $250 \mathrm{mg}$ DHA+EPA. ${ }^{2}$ Furthermore, an analysis of LC omega-3 dietary intake from NHANES 2003-2014 reported that younger individuals and women may be particularly at risk: the mean EPA+DHA intake for children $1-5$ years was just $23.1 \mathrm{mg} / 1000 \mathrm{kcal}$, which is below the DGA recommendation for EPA+DHA. ${ }^{17} \mathrm{~A}$ further study of NHANES 2001-2014 reported that more than $95 \%$ of women of childbearing age did not meet the DGA recommendations for EPA+DHA. ${ }^{14}$

Collectively, the dietary intake data from NHANES as well as other populations ${ }^{18}{ }^{19}$ provide compelling evidence of gaps in LC omega-3 intake. However, dietary intake is limited by measurement error. ${ }^{20}$ Use of errorprone dietary assessment tools can lead to spurious findings, contributes to heterogeneity in evidence on diet and health and has led some to be critical of self-reported dietary data being used to inform public health recommendations. ${ }^{21}$ Biomarkers of dietary intake (ie, circulating omega-3s) may provide more accurate information on LC omega-3 status and may also confirm findings from studies of dietary intake. Accurate reference range data for fatty acids are critical for identifying at-risk populations and setting appropriate public health recommendations. However, reference data on fatty acids in large, representative populations are scarce. The last data of this kind in the USA were from NHANES 2003-2004 in adults only. Therefore, the aim of this study was to provide contemporary reference ranges for LC omega-3 fatty acids in the US across all life stages using the most recent serum fatty acid data (2011-2012) available in NHANES. An additional objective was to determine the proportion of the US population with serum LC omega-3 fatty acid concentrations below concentrations equivalent to DGA intake recommendations and below concentrations associated with cardioprotection.

\section{MATERIALS AND METHODS}

This study uses the NHANES 2011-2012 public-use data files. NHANES is designed to assess the nutritional status and health of children and adults in the USA, using a complex multistage probability sampling design that is representative of the national civilian population in the USA. ${ }^{22}$ Fatty acids were measured in serum with the goal of obtaining US reference ranges for most circulating fatty acids in a subsample of participants. The subsample was selected to be a nationally representative sample. Participants were chosen at random from all participants, age 3-11 who attended mobile examinations and fasting participants aged 12 and older who were examined in the morning session. Of the 5643 participants in the subsample, 397 participants did not have a lab specimen. Fatty acids were measured using modified methods of Lagerstedt $e t a l .{ }^{23}$ Briefly, total fatty acids were hexane extracted along with an internal standard solution for fatty acid recovery. The extract was derivatised to form pentafluorobenzyl esters and injected onto a capillary gas chromatograph column. A total of 30 dietary fatty acids were quantitated. The lower limit of detection for EPA, DHA and docosapentaenoic acid (DPA) was $0.79 \mu \mathrm{mol} / \mathrm{L}, 1.84$ $\mu \mathrm{mol} / \mathrm{L}$ and $0.24 \mu \mathrm{mol} / \mathrm{L}$, respectively. Fatty acids were expressed as a percent of total fatty acids (respective fatty acid/sum of 30 fatty acids measured) in this analysis to facilitate comparison with other studies, which predominately report fatty acids as a relative percent. ${ }^{24-26}$ Missing data (not including those with values below the limit of detection) for a given fatty acid ranged from a low of $\mathrm{N}=1222$ (EPA) to 1861 (eicosatrienoic acid). As relative fatty acids require a common denominator of total fatty acids, individuals who were missing data for any fatty acid were excluded $(\mathrm{N}=2983)$, which resulted in an overall sample of 2261 participants: 945 children, age 3-19 and 1316 adults, age 20 and older.

Mean EPA, DHA and the sum of LC omega-3s (EPA+D$\mathrm{PA}+\mathrm{DHA})$ were calculated for children and adults as well as by key life stages: early childhood (3-5 years), middle childhood (6-11 years), adolescents (12-19 years), adults (20-55), seniors ( $>55)$ and women who were pregnant and/or breastfeeding. Pregnancy status was determined using the variable pregnancy status at examination (RIDEXPREG) from the demographics data and the variable pregnancy test (URXPREG) from the laboratory data. Breastfeeding status was determined from the reproductive health data (RHQ200). Women of childbearing age were defined as those 15-44 years of age, which corresponded to the age range of women who reported being pregnant and/or breastfeeding. Each life stage was also analysed by gender and race/ethnicity. Supplements containing fish oil, cod liver oil, salmon oil, krill oil and DHA were referred to in this analysis as 'LC omega-3 supplements' and were identified from participants report of dietary supplement use in the past 30 days.

Serum LC omega-3 fatty acids reflect short-term (weeks to months) intake of dietary fat. ${ }^{27} 28$ To provide additional public health context to findings, comparisons were made to fatty acid concentrations (LC omega-3s $=2.49 \%$ ), previously identified as approximately equivalent to DGA dietary recommendations of $250 \mathrm{mg} /$ day of EPA and DHA. ${ }^{29}$ Comparisons were also made to serum fatty 
acid concentrations associated with cardioprotection. Virtanen $e t a l^{31}$ reported lower risk of atrial fibrillation; a common cardiac arrhythmia among participants with serum DHA $\geq 2.85 \%$. Reduced risk of mortality, sudden cardiac arrest and other adverse cardiac events have been consistently associated with higher red blood cell (RBC) concentrations of EPA+DHA, also known as the Omega-3 Index. ${ }^{32}$ To compare serum NHANES data with the Omega-3 Index, an equation relating the RBC-based metric with plasma EPA+DHA concentrations was applied to calculate risk categories for cardiovascular disease. ${ }^{33} 34$ This equation was chosen based on previous evidence that the fatty acid composition of serum and plasma is similar. $^{35}$ Application of the equation resulted in the following Omega-3 Index categories: low risk $>6.23 \%$, intermediate risk $3.11 \%-6.23 \%$ or high risk $<3.11 \%$.

The following covariates were used to characterise the sample population: age, gender, race/ethnicity (NonHispanic White, Hispanic/Mexican-American, NonHispanic Black, Asian and other/unknown), and for adults: poverty income ratio (PIR; low: $0-1.85$, medium: $>1.85-3.50$ and high: $>3.50$ ), education (< high school, high school and $>$ high school), current smoking status (yes/no) and body mass index (BMI).

Sample sizes in the text and tables are unweighted. However, estimates for means, proportions, SE and 95\% CIs were weighted using sampling weights for the fatty acid subsample. Variance estimate was determined using the Taylor series linearisation approach. Differences between groups were determined by linear regression at $\mathrm{p}<0.05$. Statistical analyses were performed using survey procedures in STATA software V.14.2 (StataCorp, College Station, Texas, USA).

\section{Patient and public involvement}

Patients and the public were not directly involved in the design, conduct, reporting or dissemination of this study.

\section{RESULTS}

\section{Demographic characteristics}

A total of 2261 participants were included (table 1), $47.6 \%$ were men, $52.5 \%$ were women; $41.8 \%$ were $3-19$ years of age; $58.2 \%$ were 20 years of age and older; $16.5 \%$ were Mexican American or Hispanic, $65.2 \%$ were nonHispanic white, $12.1 \%$ were non-Hispanic black and $4.55 \%$ were Asian. With respect to education, $5.36 \%$ had education below high school, $11.2 \%$ had a high school education, $63.7 \%$ had some college or higher education and $19.7 \%$ had education in between (more than high school). Among adults, 20.2\% were current smokers; 9.90\% had low PIR, 32.7\% had medium PIR and $66.3 \%$ had high PIR. The mean (SE) BMI among adults was 28.7 $(0.34) \mathrm{kg} / \mathrm{m}^{2}$. LC omega-3 supplement use was low, with only $7.3 \%(\mathrm{~N}=166)$ in the overall population reporting use in the prior 30 days.

\section{Mean concentrations of omega-3 fatty acids}

Mean serum concentrations of EPA, DHA and LC omega-3s by age group are shown in table 2. Among children 3-19
Table 1 Characteristics of study participants in the NHANES 2011-2012 fatty acid sample

\begin{tabular}{|c|c|c|}
\hline Characteristics & & N (\%) \\
\hline \multirow[t]{2}{*}{ Gender } & Male & $1098(47.6)$ \\
\hline & Female & $1163(52.5)$ \\
\hline \multirow[t]{7}{*}{ Age group } & Children, 3-19years & $945(41.8)$ \\
\hline & Adults, 20 years+ & $1316(58.2)$ \\
\hline & $\begin{array}{l}\text { Early childhood, } \\
3-5 \text { years }\end{array}$ & $179(2.40)$ \\
\hline & $\begin{array}{l}\text { Middle childhood, } \\
6-11 \text { years }\end{array}$ & $519(6.52)$ \\
\hline & $\begin{array}{l}\text { Adolescents, 12- } \\
19 \text { years }\end{array}$ & $247(10.3)$ \\
\hline & Adults, 20-55years & $818(53.9)$ \\
\hline & Seniors, $>55$ years & $498(26.9)$ \\
\hline \multirow[t]{5}{*}{ Race/ethnicity } & $\begin{array}{l}\text { Mexican American/ } \\
\text { Hispanic }\end{array}$ & $605(16.5)$ \\
\hline & Non-Hispanic White & $729(65.2)$ \\
\hline & Non-Hispanic Black & $592(12.1)$ \\
\hline & Asian & $265(4.55)$ \\
\hline & Other/unknown & $70(1.66)$ \\
\hline \multirow[t]{4}{*}{ Education adults } & $<$ High school & $121(5.36)$ \\
\hline & High school & $182(11.2)$ \\
\hline & $>$ High school & $279(19.7)$ \\
\hline & Some college or above & $734(63.7)$ \\
\hline \multirow[t]{2}{*}{ Current smoking } & Yes & $265(20.2)$ \\
\hline & No & $1049(79.8)$ \\
\hline \multirow[t]{3}{*}{ Poverty income ratio } & Low 0-1.85 & $20(9.90)$ \\
\hline & Medium >1.85-3.50 & $423(32.7)$ \\
\hline & High $>3.50$ & $712(66.3)$ \\
\hline BMI adults & Mean, $\mathrm{kg} / \mathrm{m}^{2}$ & $28.7(0.34)$ \\
\hline $\begin{array}{l}\text { LC omega-3 } \\
\text { supplement use, N } \\
(\%)\end{array}$ & Yes & $166(7.30)$ \\
\hline
\end{tabular}

Sample sizes are unweighted, however, estimates for means, proportions and standard errors are weighted. Poverty to income ratio is defined as total family income relative to poverty guidelines. Long-chain (LC) omega-3 supplement use defined from selfreported supplement use (fish oil, cod liver oil, salmon oil, krill oil and $\mathrm{DHA}$ ) in the prior 30 days.

BMI, body mass index; NHANES, National Health and Nutrition Examination Survey.

years, mean (SE) EPA, DHA and LC omega-3s were $0.28 \%$ $(0.01), 1.07 \%(0.02)$ and $1.75 \%(0.03)$, respectively. Among adults 20 and older, mean (SE) EPA, DHA and the sum of LC omega-3s were $0.61 \%(0.02), 1.38 \%(0.05)$ and $2.43 \%(0.08)$, respectively. Concentrations of EPA, DHA and the sum of LC omega-3s were all significantly lower $(\mathrm{p}<0.001)$ in children compared with adults. Mean (SE) concentrations of EPA, DHA and the sum of LC omega-3s in early childhood were $0.26 \%(0.01), 1.01 \%$ (0.03), $1.65 \%$ (0.04); middle childhood: $0.29 \%$ (0.02), 
Table 2 Mean serum LC omega-3 fatty acid concentrations (\% of total fatty acids) by age group in NHANES 2011-2012

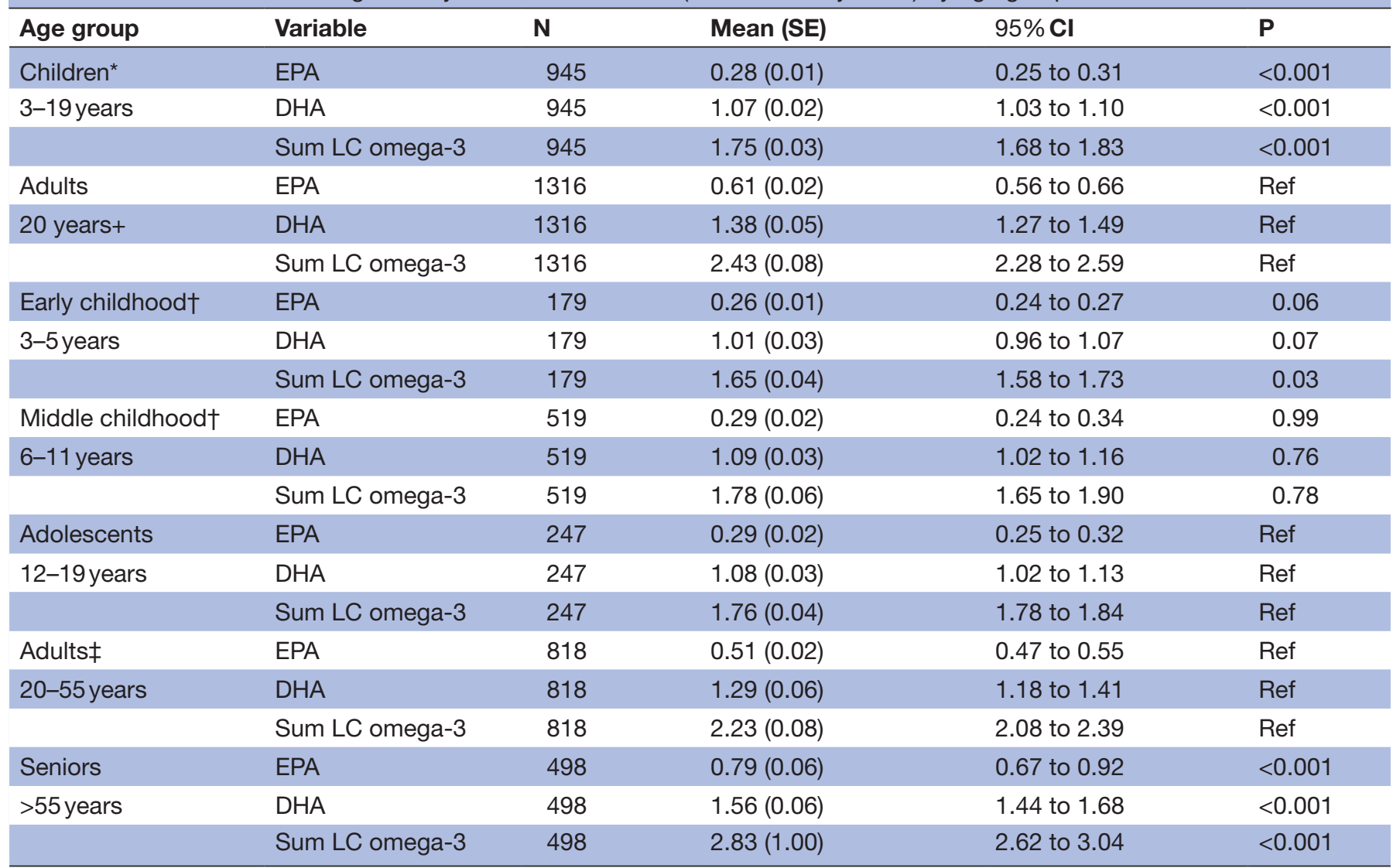

Comparisons are *children 3-19 years vs adults 20+years, †early childhood and middle childhood vs adolescents, and $\ddagger$ adults vs seniors. Sum LC omega-3 represents EPA+DPA+DHA.

BMI, body mass index; DHA, docosapentaenoic acid; EPA, eicosapentaenoic acid; LC, long chain; NHANES, National Health and Nutrition Examination Survey.

$1.09 \%(0.03), 1.78 \%(0.06)$ and adolescents: $0.29 \%$ $(0.02), 1.08 \%(0.03)$ and $1.76 \%(0.04)$. Compared with adolescents, the sum of LC omega-3s was lower in early childhood $(p=0.03)$. Similarly, EPA and DHA tended to be lower in early childhood $(p=0.06$ and $p=0.07)$. Mean (SE) concentrations of EPA, DHA and the sum of LC omega-3s in adults 20-55 years old were $0.51 \%(0.02)$, $1.29 \%$ (0.06), $2.23 \%$ (0.08), which were significantly lower than in seniors (all p<0.001): $0.79 \%(0.06), 1.56 \%(0.06)$, $2.83 \%(1.00)$. The prevalence of LC omega-3 supplement use ranged from low use $\mathrm{N}=3(1.21 \%)$ in adolescents to high use $\mathrm{N}=84$ (16.9\%) in seniors.

Mean serum concentrations of EPA, DHA and the sum of LC omega-3s by life stage and gender are shown in online supplemental table 1 and by race/ethnicity among children and adults in online supplemental table 2. Among adults 20 and older, men had significantly lower serum concentrations of EPA $(p=0.01)$, DHA $(p<0.001)$ and the sum of LC omega-3s $(p<0.001)$ compared with women. When further examined by life stage, men consistently had lower concentrations beginning with adolescent men having significantly lower DHA concentrations than women $(\mathrm{p}=0.03)$. Additionally, men aged 20-55 and men $>55$ had significantly lower DHA and sum of LC omega-3 fatty acids than women of 20-55 ( $\mathrm{p}=0.001$ and $\mathrm{p}=0.01)$ and women $>55 \quad(\mathrm{p}=0.03$ and $\mathrm{p}=0.04)$. Race/ ethnicity differences between omega-3s were similar among children and adults: relative to non-Hispanic white individuals, EPA was significantly lower in Mexican American/Hispanic individuals, DHA was significantly lower in non-Hispanic black individuals, and EPA, DHA and the sum of LC omega-3s were all significantly higher among Asian individuals. Mean concentrations of EPA, DHA and LC omega-3s are also shown in online supplemental table 3 by Institute of Medicine age categories to facilitate comparison with dietary recommendations. Reference concentrations of all 27 fatty acids additionally measured in the NHANES 2011-2012 cycle are shown in online supplemental table 4 .

Mean (SE) serum concentrations of EPA, DHA and the sum of LC omega-3s by pregnancy/breastfeeding status are shown in table 3 . There was no statistical difference in the concentrations of EPA among pregnant/breastfeeding women compared with among women of childbearing age $(p=0.17)$. DHA and the sum of LC omega-3s among pregnant/breastfeeding women who were not statistically different than those in women of childbearing age. LC omega-3 supplement use was reported by two 
Table 3 Mean serum LC omega-3 fatty acid concentrations (\% of total fatty acids) in women who were pregnant/ breastfeeding $(\mathrm{N}=21)$ and women of childbearing age $(\mathrm{N}=319)$

\begin{tabular}{|c|c|c|c|c|c|}
\hline \multirow[b]{2}{*}{ Fatty acid } & \multirow{2}{*}{$\begin{array}{l}\text { Pregnant/breastfeeding } \\
\text { Mean (SE) } \\
\end{array}$} & \multirow[b]{2}{*}{$95 \% \mathrm{Cl}$ for mean } & \multirow{2}{*}{$\begin{array}{l}\text { Women of childbearing age } \\
\text { Mean (SE) } \\
\end{array}$} & \multirow[b]{2}{*}{$95 \% \mathrm{Cl}$ for mean } & \multirow[b]{2}{*}{$\mathbf{P}$} \\
\hline & & & & & \\
\hline EPA & $0.34(0.07)$ & 0.19 to 0.49 & $0.47(0.03)$ & 0.39 to 0.53 & 0.17 \\
\hline Sum LC omega-3 & $2.18(0.15)$ & 1.87 to 2.49 & $2.24(0.10)$ & 2.02 to 2.46 & 0.73 \\
\hline
\end{tabular}

Sample sizes are unweighted, however, estimates for means and SE are weighted. Women of childbearing age are those, age 15-44 years. DPA, docosapentaenoic acid; EPA, eicosapentaenoic acid; LC, long chain.

(9.52\%) pregnant/breastfeeding women compared with $21(6.03 \%)$ women of childbearing age.

The prevalence of individuals with concentrations below those approximately equivalent to the DGA recommendations is shown in table 4 by gender and life stage. Overall, $95.5 \%$ of children and $68.3 \%$ of adults age 20 years and older had LC omega-3 concentrations below $2.49 \%$, corresponding to intake of LC omega-3 recommended by the DGA. The prevalence of those below the DGA recommendations ranged from $97.5 \%$ of women in early childhood to $52.3 \%$ of women seniors. Early childhood (age 3-5 years), middle childhood (age 6-11 years and adolescents (age 12-19 years), had the largest prevalence of low LC omega-3 concentrations, with $97.4 \%$, $94.8 \%$ and $95.5 \%$ of the population in these age groups below LC omega-3 concentrations $(<2.49 \%)$ recommended by the DGA, respectively. Figure 1 depicts the prevalence of participants with serum DHA and Omega-3 Index in previously established risk categories. ${ }^{31} 33$ The vast majority of adults had DHA concentrations below that associated with lower risk of atrial fibrillation (2.85\%): $97 \%$ of all adults, $98 \%$ of men and $96.2 \%$ of women. Among all adults, $88.7 \%$ of the population had an Omega-3 Index in the high cardiovascular risk category, $10.4 \%$ of the adults were in the intermediate risk category and just $1.0 \%$ of the adults were in the low risk category. The prevalence of those with an Omega-3 Index in the low cardiovascular risk category ranged from a low of $0.34 \%$ of adult men to a high of $3.08 \%$ among senior women (online supplemental table 5).

\section{DISCUSSION}

This study is one of the few to provide information on reference concentrations of serum LC omega-3 (a biomarker of omega-3 nutritional status) in a large, nationally representative sample of Americans from early childhood into later life. This is critical for providing benchmarking data on omega-3 status and tracking progress (or lack thereof) towards guidance from expert committees to increase intake and for the general public to consume 8 ounces per week of a variety of seafood, providing approximately $250 \mathrm{mg}$ of EPA and DHA per day. ${ }^{2}$ The mean EPA and DHA serum concentrations of $0.28 \%$ and $1.07 \%$ in children (3-19 years), and $0.61 \%$ and $1.38 \%$ in adults (20 years+), reflects the persistently low consumption of seafood and omega-3s. Using estimates of LC omega-3 concentrations approximately equivalent to the DGA dietary intake recommendations for EPA and DHA, nearly all children in the study, and over $68 \%$ of adults had serum LC omega-3 concentrations below those associated with US Dietary recommendations for LC omega-3 consumption. When further examined by life stage and demographics, several potentially vulnerable populations emerged that may warrant targeted promotion of EPA and DHA consumption; specifically early childhood (ages 3-5 years), adult men, Mexican American/Hispanic individuals and non-Hispanic black individuals tended to have particularly low serum concentrations of LC omega-3 fatty acids. Pregnant and/or breastfeeding women also appear to be a population of concern as LC omega-3 fatty acid concentrations were similar to women of comparable

Table 4 Prevalence, N (\%) of EPA+DHA fatty acid concentrations $<2.49 \%$, which is approximately equivalent to DGA dietary recommendations for EPA and DHA intake by gender and age group in NHANES 2011-2012

\begin{tabular}{|c|c|c|c|c|c|c|c|}
\hline & Children, 3-19years & $\begin{array}{l}\text { Adults, } 20 \\
\text { years+ }\end{array}$ & Early childhood & Middle childhood & Adolescents & Adults & Seniors \\
\hline & \multicolumn{7}{|l|}{$<2.49 \%$ EPA+DHA } \\
\hline Male & 429 (96.6) & 445 (72.4) & 85 (97.3) & $239(94.7)$ & 105 (97.6) & $313(80.7)$ & $132(53.7)$ \\
\hline Female & $451(94.5)$ & $411(64.6)$ & $83(97.5)$ & $249(94.9)$ & 119 (93.6) & $280(71.4)$ & $131(52.3)$ \\
\hline
\end{tabular}

DGA, Dietary Guidelines for Americans; DHA, docosapentaenoic acid; EPA, eicosapentaenoic acid; NHANES, National Health and Nutrition Examination Survey. 


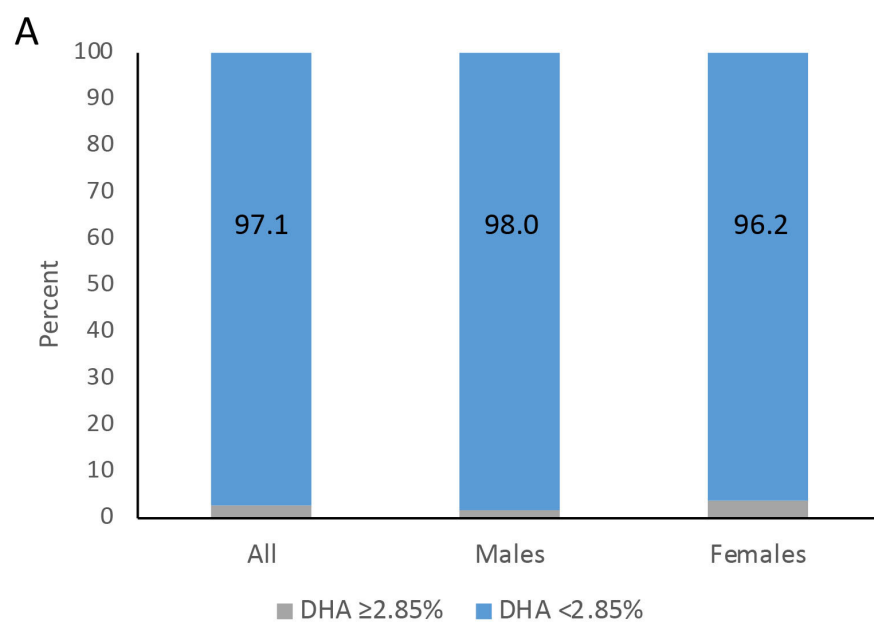

B

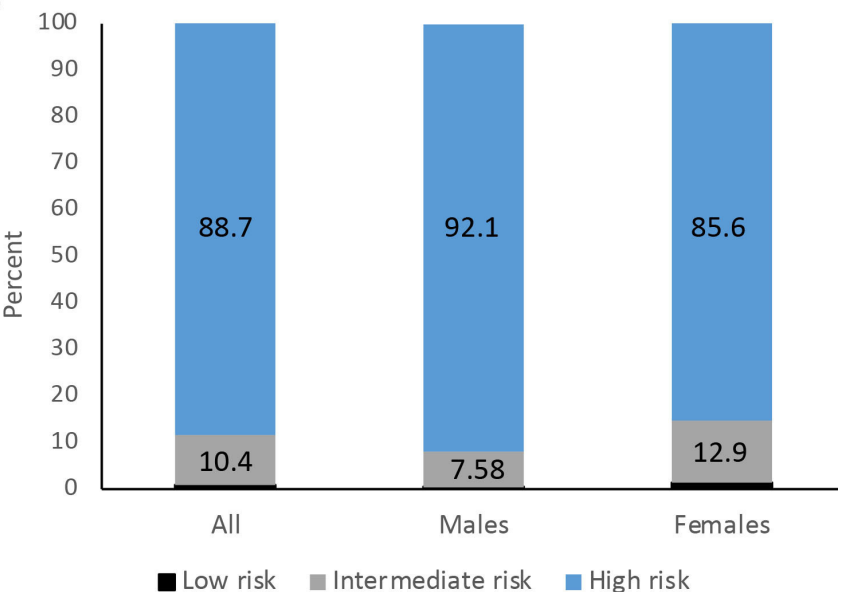

Figure 1 Comparison to long-chain omega-3 concentrations from prior studies were used to approximate (A) the prevalence of participants with omega-3 concentrations equivalent to omega-3 dietary intake recommendations by the $\mathrm{DGA},{ }^{30}(\mathrm{~B})$ the lower range of the tertiles of serum DHA associated with lower risk of atrial fibrillation ${ }^{31}$ and (3) cardiovascular risk categories defined by the Omega-3 Index. ${ }^{33} 34$ DHA, docosapentaenoic acid; DGA, Dietary Guidelines for Americans.

age despite recommendations for higher EPA and DHA consumption to support infant development. ${ }^{2}$

Adults, particularly seniors, had the highest concentrations of serum EPA, DHA and sum of LC omega-3s, although only around one quarter of adults, and approximately half of seniors, had concentrations corresponding to DGA consumption recommendations. However, these concentrations are not adequate for cardioprotection; less than $2 \%$ of seniors had an Omega-3 Index in the low cardiovascular disease risk category. The notably higher serum concentrations of LC omega-3s in seniors $>55$ relative to adults $20-55$ years mirrors findings from previous studies of LC omega-3 dietary intake $^{1517}$ that report higher intake and also greater use of omega-3 supplementation among seniors. Currently, there is no established Adequate Intake (AI) for EPA or DHA; the AI for alpha-linolenic acid for men and women is the same for those age 14 and older. ${ }^{36}$ However, epidemiological and physiological evidence suggests that older adults have an increased LC omega-3 requirement due to the ageing body, including brain atrophy and cognitive ageing, ${ }^{3738}$ mobility, bone health and sarcopenia. ${ }^{39-41}$ Despite LC omega-3 concentrations being highest in seniors, the implications with respect to adequacy to support the normal physiological processes underlying healthy ageing are unclear.

Our findings suggest similar DHA and sum of LC omega-3 fatty acids between women who were pregnant and/or breastfeeding and women of reproductive age. Both groups have potential implications for maternal health as well as for fetal and infant development. Omega-3s, specifically DHA, are important for fetal and infant neuron and retinal membrane development in late pregnancy and early life, essentially the first 1000 days of life. ${ }^{924}{ }^{43}$ Omega-3 intake requirements in pregnancy have not been established, however, recommendations from a number of international organisations have been put forth ${ }^{14}$ and public health messaging on the importance of LC omega-3s intake in maternal health has been promoted in recent years. ${ }^{13}$ DHA demands during pregnancy are higher due to diversion to the fetus, especially in the last trimester as the nervous system is rapidly developing, as well as expanded maternal cell mass and general requirements of the placenta. ${ }^{44}$ Pregnant women can become low in DHA if dietary intakes do not compensate for increased demands. Public health messaging has encouraged women to increase LC omega-3 intake, especially DHA through a variety of sources (low-mercury fish, and DHA found in supplements and fortified foods) ${ }^{10}$ However, the prevalence of LComega-3 supplementation in pregnant/breastfeeding women was just 9.5\%. In addition, it was found that serum EPA tended to be lower among pregnant/breastfeeding women, although not statistically significant, possibly reflecting the small sample size. This may have relevance for maternal health as epidemiologic evidence suggests that LC omega-3s plays a role in prevention and/or treatment of neuropsychiatric diseases. ${ }^{45}$ In recent years, a number of systematic reviews and meta-analyses have concluded that LC omega-3 supplementation with a higher EPA to DHA ratio, with at least $1 \mathrm{~g}$ of EPA, in conjunction with antidepressive therapy, may be more beneficial than either monotherapy alone. ${ }^{46-48}$ Associations with postpartum depression specifically are unclear, but warrant further investigation considering the prevalence of postpartum depression. ${ }^{49}$ The estimates for pregnant and breastfeeding women, met analytic and reporting criteria for NHANES, ${ }^{22}$ but nonetheless, the sample size was small and caution is warranted for interpretation of the findings. The findings are, however, consistent with a study of EPA and DHA intake in NHANES, which have pooled the samples across several cycles and found EPA and DHA intake in pregnant women did not differ from nonpregnant women in terms of being generally inadequate. ${ }^{17}$

A strength of this study is the measurement of LC omega-3s in serum rather than estimates from dietary intake, which minimises error from self-reporting. However, serum fatty acids represent short-term dietary consumption ${ }^{27} 28$ and the results may, therefore, not reflect longer term status. The large population allowed us to examine reference concentrations for multiple life stages and demographic 
categories which was weighted to be nationally representative of the USA, although sample sizes for some subgroups, for example, pregnant and breastfeeding women were limited as described above. The present study provides the most contemporaneous data in the US nationally representative population, but 8-9 years has elapsed between biospecimen collection and the present study since the NHANES serum fatty acid data only became publicly available in 2020. It is, therefore, possible that concentrations reported herein differ from those currently in the USA. Data on dietary intake of EPA and DHA in pregnant women and women of childbearing age in NHANES 2001-2014 suggest slight increases in intake across 2-year survey periods. ${ }^{14}$ Unfortunately, it is not possible to assess trends in circulating LC omega-3 fatty acids as NHANES infrequently collects circulating fatty acid data. Fatty acids were last measured in plasma collected in the 2003-2004 NHANES cycle, which captured a different number of fatty acids (24 vs 30 in 2011-2012) and was limited to individuals age 20 and older. ${ }^{29}$ The absence of standardised guidelines or definitions of adequate or optimal serum LC omega-3 concentrations to support overall health also precluded our ability to provide inferences from these results. Further limitations include that Omega-3 Index risk categories were determined from an equation relating concentrations in RBCs to plasma, which may introduce bias in our estimates and the cross-sectional nature of NHANES limits the ability to study possible causal relationships and their resultant physiological function. Finally, serum concentrations of LC omega-3 fatty acids predominately reflect exogenous dietary intake due to very limited conversion of short-chain omega-3 fatty acids to LC omega-3 fatty acids. ${ }^{50}$ However, polymorphisms that affect desaturase enzymes that convert alpha-linolenic acid to LC omega-3s may also influence circulating LC omega-3 concentrations. The presence (or absence) of such polymorphisms may confound differences in LC omega-3 between demographic groups.

\section{Conclusions}

In conclusion, this study provides reference ranges on serum LC omega-3 fatty acids, a biomarker of status, across all life stages in a nationally representative population, information that until now, was critically lacking. The findings demonstrate overall low serum concentrations of LC omega-3 fatty acids including both EPA and DHA and the sum of LC omega-3 fatty acids across all life stages. This data are supported by findings from previous dietary intake analyses. ${ }^{14-16}$ Owing to the role of LC omega-3s in brain development, there is particular need for public health strategies aimed at increasing LC omega-3 intake in children and women of childbearing age including those who are pregnant, breastfeeding or looking to become pregnant. Based on life stages, gender and demographic factors, we demonstrate that children, males, Mexican American/Hispanic and non-Hispanic Black individuals have lower EPA and/or DHA serum concentrations than adults, females and non-Hispanic white individuals, respectively. This further demonstrates the need for public health awareness and action. LC omega-3 fatty acids play a critically important role in many aspects of human health across the lifespan. Healthcare practitioners need to ensure their patients are consuming enough LC omega-3 fatty acids in their daily diet, and if they are not, they need to consider the role of supplementation for their patients to ensure nutrient gaps are being met. Furthermore, there is an increased need for guidance and education for healthcare practitioners to ensure they are prepared to make recommendations needed that include specific ways to increase EPA and DHA intake for the entire US population, with particular attention to vulnerable populations.

\section{Twitter Rachel A Murphy @RaAMurphy}

Contributors RAM, KM, PPD, SE and SHM were involved in study conception and design and advised on interpretation of the data. RAM compiled the dataset and performed the statistical analysis. RAM drafted the manuscript. KM, PPD, SE and SHM review the manuscript, provided amendment and approved the final version. RAM had full access to study data and takes responsibility for its accuracy and the integrity of the analysis.

Funding The authors have not declared a specific grant for this research from any funding agency in the public, commercial or not-for-profit sectors.

Competing interests RAM carried out the work presented in the manuscript as a consultant for Pharmavite. PPD, SE, KM and SHM are employees of Pharmavite, LLC, manufacturers and suppliers of omega-3 nutritional lipids.

\section{Patient consent for publication Not required.}

Ethics approval The NCHS Ethics Review Board protects the rights and welfare of NHANES participants. The NHANES protocol complies with the US Department of Health and Human Services' Policy for Protection of Human Research Subjects. NCHS IRB/ERC Protocol number: 2011-17. This study was exempt from the Institutional Review Board at the University of British Columbia related to the use of publicly available data for research and publication.

Provenance and peer review Not commissioned; externally peer reviewed.

Data availability statement Data are available in a public, open access repository. Data are available in a public, open access repository. The dataset used for this study was generated from data publicly released by the National Health and Nutrition Examination Survey (NHANES).

Supplemental material This content has been supplied by the author(s). It has not been vetted by BMJ Publishing Group Limited (BMJ) and may not have been peer-reviewed. Any opinions or recommendations discussed are solely those of the author(s) and are not endorsed by BMJ. BMJ disclaims all liability and responsibility arising from any reliance placed on the content. Where the content includes any translated material, BMJ does not warrant the accuracy and reliability of the translations (including but not limited to local regulations, clinical guidelines, terminology, drug names and drug dosages), and is not responsible for any error and/or omissions arising from translation and adaptation or otherwise.

Open access This is an open access article distributed in accordance with the Creative Commons Attribution Non Commercial (CC BY-NC 4.0) license, which permits others to distribute, remix, adapt, build upon this work non-commercially, and license their derivative works on different terms, provided the original work is properly cited, appropriate credit is given, any changes made indicated, and the use is non-commercial. See: http://creativecommons.org/licenses/by-nc/4.0/.

ORCID iD

Rachel A Murphy http://orcid.org/0000-0003-4383-5641

\section{REFERENCES}

1 American Heart Association. Fish and omega-3 fatty acids. Available: https://www.heart.org/en/healthy-living/healthy-eating/eat-smart/ fats/fish-and-omega-3-fatty-acids [Accessed 25 Mar 2020].

2 Dietary Guidelines Advisory Committee. Scientific report of the 2020 dietary guidelines Advisory Committee, Advisory report to the Secretary of agriculture and the Secretary of health and human services. Washington, DC: US Department of Agriculture, Agricultural Research Service, 2020. https://www.dietaryguidelines.gov/sites/ default/files/2020-07/ScientificReport_of_the_2020DietaryGuideline sAdvisoryCommittee_first-print.pdf 
3 Bowen KJ, Harris WS, Kris-Etherton PM. Omega-3 fatty acids and cardiovascular disease: are there benefits? Curr Treat Options Cardiovasc Med 2016;18:69.

4 Djoussé L, Gaziano JM, Buring JE, et al. Dietary omega-3 fatty acids and fish consumption and risk of type 2 diabetes. Am J Clin Nutr 2011;93:143-50.

5 Kaushik M, Mozaffarian D, Spiegelman D, et al. Long-chain omega-3 fatty acids, fish intake, and the risk of type 2 diabetes mellitus. Am J Clin Nutr 2009;90:613-20.

6 Wang DD, Li Y, Chiuve SE, et al. Association of specific dietary fats with total and cause-specific mortality. JAMA Intern Med 2016;176:1134-45

7 Liu JC, Conklin SM, Manuck SB, et al. Long-chain omega-3 fatty acids and blood pressure. Am J Hypertens 2011;24:1121-6.

8 Ueshima $\mathrm{H}$, Stamler J, Elliott P, et al. Food omega-3 fatty acid intake of individuals (total, linolenic acid, long-chain) and their blood pressure: INTERMAP study. Hypertension 2007;50:313-9.

9 Jensen CL. Effects of $\mathrm{n}-3$ fatty acids during pregnancy and lactation. Am J Clin Nutr 2006;83:1452S-7.

10 Greenberg JA, Bell SJ, Ausdal WV. Omega-3 fatty acid supplementation during pregnancy. Rev Obstet Gynecol 2008;1:162-9.

11 Gelenberg AJ, Freeman MP, Markowitz JC. Practice guideline for the treatment of patients with major depressive disorder. 3rd edn 2010: 51-92. http://psychiatryonline.org/pb/assets/raw/sitewi de/ practice guidelines/guidelines/mdd.pdf

12 Rimm EB, Appel LJ, Chiuve SE, et al. Seafood long-chain n-3 polyunsaturated fatty acids and cardiovascular disease: a science Advisory from the American heart association. Circulation 2018;138:e35-47.

13 WHO. Joint FAO/WHO expert consultation on fats and fatty acids in human nutrition: report of an expert consultation. Rome, Italy: Food and Agriculture Organization, 2010.

14 Zhang Z, Fulgoni V, Kris-Etherton P, et al. Dietary intakes of EPA and DHA omega- 3 fatty acids among US childbearing-age and pregnant women: an analysis of NHANES 2001-2014. Nutrients 2018;10:416. doi:10.3390/nu10040416

15 Richter CK, Bowen KJ, Mozaffarian D, et al. Total long-chain n-3 fatty acid intake and food sources in the United States compared to recommended intakes: NHANES 2003-2008. Lipids 2017;52:917-27.

16 Papanikolaou Y, Brooks J, Reider C, et al. U.S. adults are not meeting recommended levels for fish and omega-3 fatty acid intake: results of an analysis using observational data from NHANES 20032008. Nutr J 2014:13:31.

17 Thompson M, Hein N, Hanson C, et al. Omega-3 fatty acid intake by age, gender, and pregnancy status in the United States: National health and nutrition examination survey 2003-2014. Nutrients 2019;11:177.

18 Villegas R, Takata Y, Murff H, et al. Fish, omega-3 long-chain fatty acids, and all-cause mortality in a low-income US population: results from the southern community cohort study. Nutr Metab Cardiovasc Dis 2015;25:651-8.

19 Berry JD, Prineas RJ, van Horn L, et al. Dietary fish intake and incident atrial fibrillation (from the women's health Initiative). $\mathrm{Am} \mathrm{J}$ Cardiol 2010;105:844-8.

20 Thompson FE, Kirkpatrick SI, Subar AF, et al. The National cancer Institute's dietary assessment primer: a resource for diet research. $J$ Acad Nutr Diet 2015;115:1986-95.

21 Archer E. The NHANES dietary data are physiologically implausible and inadmissible as scientific evidence. Am J Clin Nutr 2017;106:951-2.

22 Johnson CL, Paulose-Ram R, Ogden CL. National health and nutrition examination survey: analytic guidelines, 1999-2010. Vital Health Stat 2013;161:1-24.

23 Lagerstedt SA, Hinrichs DR, Batt SM, et al. Quantitative determination of plasma c8-c26 total fatty acids for the biochemical diagnosis of nutritional and metabolic disorders. Mol Genet Metab 2001;73:38-45

24 Sun Y, Koh HWL, Choi H, et al. Plasma fatty acids, oxylipins, and risk of myocardial infarction: the Singapore Chinese Health study. J Lipid Res 2016;57:1300-7.

25 Wu JHY, Marklund M, Imamura F, et al. Omega-6 fatty acid biomarkers and incident type 2 diabetes: pooled analysis of individual-level data for 39740 adults from 20 prospective cohort studies. Lancet Diabetes Endocrinol 2017;5:965-74.
26 Imamura F, Fretts A, Marklund M, et al. Fatty acid biomarkers of dairy fat consumption and incidence of type 2 diabetes: a pooled analysis of prospective cohort studies. PLoS Med 2018;15:e1002670.

27 Holman RT. Control of polyunsaturated acids in tissue lipids. J Am Coll Nutr 1986;5:183-211.

28 Kwon JS, Snook JT, Wardlaw GM, et al. Effects of diets high in saturated fatty acids, canola oil, or safflower oil on platelet function, thromboxane B2 formation, and fatty acid composition of platelet phospholipids. Am J Clin Nutr 1991;54:351-8.

29 Murphy RA, Yu EA, Ciappio ED, et al. Suboptimal plasma long chain $\mathrm{n}-3$ concentrations are common among adults in the United States, NHANES 2003-2004. Nutrients 2015;7:10282-9.

30 Sun Q, Ma J, Campos H, et al. Comparison between plasma and erythrocyte fatty acid content as biomarkers of fatty acid intake in US women. Am J Clin Nutr 2007;86:74-81.

31 Virtanen JK, Mursu J, Voutilainen S, et al. Serum long-chain n-3 polyunsaturated fatty acids and risk of hospital diagnosis of atrial fibrillation in men. Circulation 2009;120:2315-21.

32 von Schacky C. Omega-3 index and cardiovascular health. Nutrients 2014;6:799-814.

33 Harris WS. The omega-3 index as a risk factor for coronary heart disease. Am J Clin Nutr 2008;87:1997S-2002.

34 Chaudhary R, Saadin K, Bliden KP, et al. Risk factors associated with plasma omega-3 fatty acid levels in patients with suspected coronary artery disease. Prostaglandins Leukot Essent Fatty Acids 2016;113:40-5.

35 Armstrong JM, Metherel AH, Stark KD. Direct microwave transesterification of fingertip prick blood samples for fatty acid determinations. Lipids 2008:43:187-96.

36 Panel on Macronutrients, Panel on the Definition of Dietary Fiber, Subcommittee on Upper Reference Levels of Nutrients. Dietary reference intakes for energy, carbohydrate, fiber, fat, fatty acids, cholesterol, protein, and amino acids. Washington, DC: National Academies Press, 2005. https://www.nap.edu/catalog/10490

37 Issa AM, Mojica WA, Morton SC, et al. The efficacy of omega-3 fatty acids on cognitive function in aging and dementia: a systematic review. Dement Geriatr Cogn Disord 2006;21:88-96.

38 Denis I, Potier B, Heberden C, et al. Omega-3 polyunsaturated fatty acids and brain aging. Curr Opin Clin Nutr Metab Care 2015:18:139-46.

39 Reinders I, Murphy RA, Song X, et al. Polyunsaturated fatty acids in relation to incident mobility disability and decline in gait speed; the age, gene/environment susceptibility-reykjavik study. Eur J Clin Nutr 2015;69:489-93.

40 Harris TB, Song X, Reinders I, et al. Plasma phospholipid fatty acids and fish-oil consumption in relation to osteoporotic fracture risk in older adults: the age, Gene/Environment susceptibility study. Am J Clin Nutr 2015;101:947-55.

41 Dupont J, Dedeyne L, Dalle S, et al. The role of omega-3 in the prevention and treatment of sarcopenia. Aging Clin Exp Res 2019;31:825-36.

42 Middleton P, Gomersall JC, Gould JF, et al. Omega-3 fatty acid addition during pregnancy. Cochrane Database Syst Rev 2018;15:CD003402.

43 Carlson SE, Gajewski BJ, Valentine CJ, et al. Assessment of DHA on reducing early preterm birth: the ADORE randomized controlled trial protocol. BMC Pregnancy Childbirth 2017;17:62.

44 Rees A-M, Austin M-P, Owen C, et al. Omega-3 deficiency associated with perinatal depression: case control study. Psychiatry Res 2009;166:254-9.

45 Larrieu T, Layé S. Food for mood: relevance of nutritional omega-3 fatty acids for depression and anxiety. Front Physiol 2018;9:1047.

46 Liao $\mathrm{Y}$, Xie B, Zhang $\mathrm{H}$, et al. Efficacy of omega-3 PUFAs in depression: a meta-analysis. Trans/ Psychiatry 2019;9:190.

47 Hallahan B, Ryan T, Hibbeln JR, et al. Efficacy of omega-3 highly unsaturated fatty acids in the treatment of depression. $\mathrm{Br} \mathrm{J}$ Psychiatry 2016;209:192-201.

48 Wojcicki JM, Heyman MB. Maternal omega-3 fatty acid supplementation and risk for perinatal maternal depression. $J$ Matern Fetal Neonatal Med 2011;24:680-6.

49 Browne JC, Scott KM, Silvers KM. Fish consumption in pregnancy and omega-3 status after birth are not associated with postnatal depression. J Affect Disord 2006;90:131-9.

50 Plourde M, Cunnane SC. Extremely limited synthesis of long chain polyunsaturates in adults: implications for their dietary essentiality and use as supplements. Appl Physiol Nutr Metab 2007;32:619-34. 